\title{
Estimating Energy Savings from Bus Improvement Options in Urban Corridors
}

Moazzem Hossain, Department of Civil Engineering, Bangladesh University of Engineering and Technology (BUET), Bangladesh Scott Kennedy, Masdar Institute of Science and Technology, UAE

\section{Abstract}

The potential to achieve significant energy savings in the road transport sector can be a powerful driver to promote bus transport, especially bus rapid transit (BRT) development. This research introduces a spreadsheet tool for making realistic estimates of energy savings due to increased use of buses, with an explicit inclusion of the effect of congestion on traffic flow and fuel consumption. Based on scenarios developed around projected growth in trip demand, changes in vehicle technology, lane expansion, and modal distribution of trips, the model determines typical daily profiles for fuel consumption by vehicle types. A case study has been performed on an urban corridor in the city of Kuala Lumpur to compare energy usage among three scenarios: business as usual, conventional bus lane, and full-scale BRT implementation. The BRT provides significant energy savings over both alternatives, with the greatest savings achieved when locating the BRT in a newly constructed lane.

\section{Introduction}

The transport sector is the world's primary consumer of petroleum products, accounting for 58 percent of global total final consumption in 2004 (IEA 2007). For developing and developed countries alike, the greatest share of this sector's 
fuel consumption comes from road transport (U.S. DOE 2007; NEB 2005; IEA 2007; UN ESCAP 2005). In light of concerns about oil price volatility, domestic energy security, and the environmental impact of burning fossil fuels, the road transport sector has been subject to increasing scrutiny over how its energy consumption can be reduced. Energy efficiency has now become a key component in recent initiatives to promote sustainable transport, particularly in the urban context. These include numerous regional and multinational efforts as well as initiatives spearheaded by individual municipalities (see examples in GDRC 2007).

Among the different tools promoted to reduce road transport energy consumption, improvement of public bus systems is commonly recognized as a costeffective option that can be implemented in the very near term (Hensher 2007). Under favorable conditions, increasing the modal share of public bus over private transport can achieve significant benefits in both reduced energy consumption and improved air quality due to the higher energy efficiency per passenger-km of bus transit (Romilly 1999; Shariar and Kahn 2003; Hossain and Kennedy 2006). Enhancements to public buses, such as increased frequency, reserved bus lanes, and full-scale bus rapid transit (BRT), can increase transit ridership as long as a supportive transport policy framework is in place. It has been claimed that highquality BRT systems that replace conventional on-street bus services should be as effective as rail-based systems in generating patronage (Graham 2005). In addition, increased modal shift in favor of public transport can result in fewer cars utilizing the same road space with a possible speed advantage and fewer flow breakdown situations. Fewer vehicles moving at a higher speed have an important bearing on the fuel consumption of the urban corridor.

To date, most analyses of energy consumption by the transport sector utilize a top-down approach that draws on fuel consumption statistics at the national or regional level. While this method can provide a gross indication of total energy demand by different modes, it cannot capture the effects of modal shift at the operational level, such as changes in traffic congestion and trip travel times. For example, a long-term energy planning tool such as LEAP (SEI 2006) can set a sectoral target of energy consumption and can estimate the energy consumption across various vehicle categories, but it cannot estimate the impact of initiatives that alter traffic flow in localized areas. Incorporating operational details and arrangements into energy consumption estimates is more significant when it comes down to a project implementation level. As an example, approval and implementation of a bus improvement project could receive a significant boost 
with a more realistic estimation of potential energy savings. Utilization of microsimulation models along with the incorporation of vehicular emission functions may prove to be highly demanding in terms of technical know-how and cost implications for many cities. This article introduces a simpler spreadsheet tool that can facilitate the estimation of potential energy savings with efficient public transport alternatives under various traffic scenarios.

Development of such a model requires information on vehicular emission and their dependence on driving behavior (i.e., instantaneous speed, acceleration, and idling). A number of research studies (Brzezinski, Enns, and Hart 1999; Biggs and Akcelik 1986; Post et al. 1984) have proposed various mobile source emission models that calculate fuel consumption as an intermediate output to determine total vehicular emissions. While some of these models incorporate realistic driving cycles, changes in travel demand, vehicle aging and other effects, the majority cannot be used to assess the energy use impact of more dynamic variables (i.e., idle time, acceleration/deceleration, etc.) that depend on vehicle operating conditions (Barth et al. 1996). A comprehensive report on emission inventory methodologies (EEA 2005) suggests two methods for including vehicle speed effects on mobile emissions. First, driving conditions can be categorized according to road type (i.e., urban, rural, or highway) and the emissions estimated based on speed-dependent emission factors and a mean vehicle speed for each category. Alternatively, speed-dependent emission functions can be integrated over speed-distribution curves that cover the entire range of driving conditions and their probability of occurrence. In the end, the authors suggest that the added complexity of including speed-distribution curves for calculating mobile source emission inventories may not be justified due to the high uncertainty in estimating vehicle emission factors. This uncertainty may result from a wide discrepancy of emission factors for vehicles of different type and age (Ntziachristos and Samaras 2000). On the other hand, in estimating $\mathrm{CO}_{2}$ emissions and fuel consumption, little variation exists among vehicles of similar engine size. In this case, including actual operating conditions in the energy estimation may be well justified. Such an effort can build from previous work to collect data on instantaneous speed-dependent fuel and emissions curves for different vehicle types (Rakha et al. 2000; Tong et al. 2000).

The present study focuses on the development of a modeling tool that includes the above issues in estimating the energy savings for bus transit improvement options. When applied to bus systems, the improvements may range from a simple demarcation of an exclusive bus lane to fully segregated high-quality BRT 
systems. With increased speed in a BRT lane, and potentially in other lanes if the number of private vehicles is reduced, BRT systems have the ability to decrease energy consumption by the transport sector significantly. However, due to technological, operational, and behavioral changes, forecasting the effect of bus system enhancements on energy consumption is not a simple task. For this reason, a decision support tool, the Sustainable Transport and Energy Planning (STEP) model, has been developed to assess the impact of such bus system implementation on the energy consumption along a defined corridor. The following section describes the modeling framework including model mechanisms, model data base, and calibrations. We then introduce transit and traffic scenarios for application of the model in a Kuala Lumpur corridor. Next, we describe the results of energy savings from model application. Discussions and implications of the results are presented in the concluding section.

\section{Modeling Framework}

The STEP model has been developed as a scenario-based planning tool to estimate the total energy consumption along a single traffic corridor for all road transport modes and vehicle types, ${ }^{1}$ with a special focus on bus transport. Scenarios are based on projections of passenger trip demand, mix of vehicle types, distribution between public and private modes, vehicle occupancy, and the number of lanes. The corridor itself is divided into sections, with each section characterized according to flow type, flow direction, number of lanes, and number of traffic signals or traffic circles. The model has two distinct parts, a traffic model and a fuel consumption calculator. For the former, projected passenger trip demand and other scenario parameters are used to determine typical daily profiles for average speed and volume along the corridor. It is advisable to construct three separate profiles for weekdays and weekends. For the latter, the fuel consumed by each vehicle category is calculated based on vehicle speed, travel time, volume, and fuel consumption curves for each vehicle type. In the case of dedicated bus lanes, volume, speed, and fuel consumption outputs are calculated separately for the bus lanes and nonbus lanes. The advantage of the present approach is that the effects of congestion and unstable vehicle flow are explicitly modeled and used in the estimations of fuel consumption and diverted demand (i.e., unmet demand diverted to an alternate route due to severe congestion). The model flow is summarized in Figure 1. 


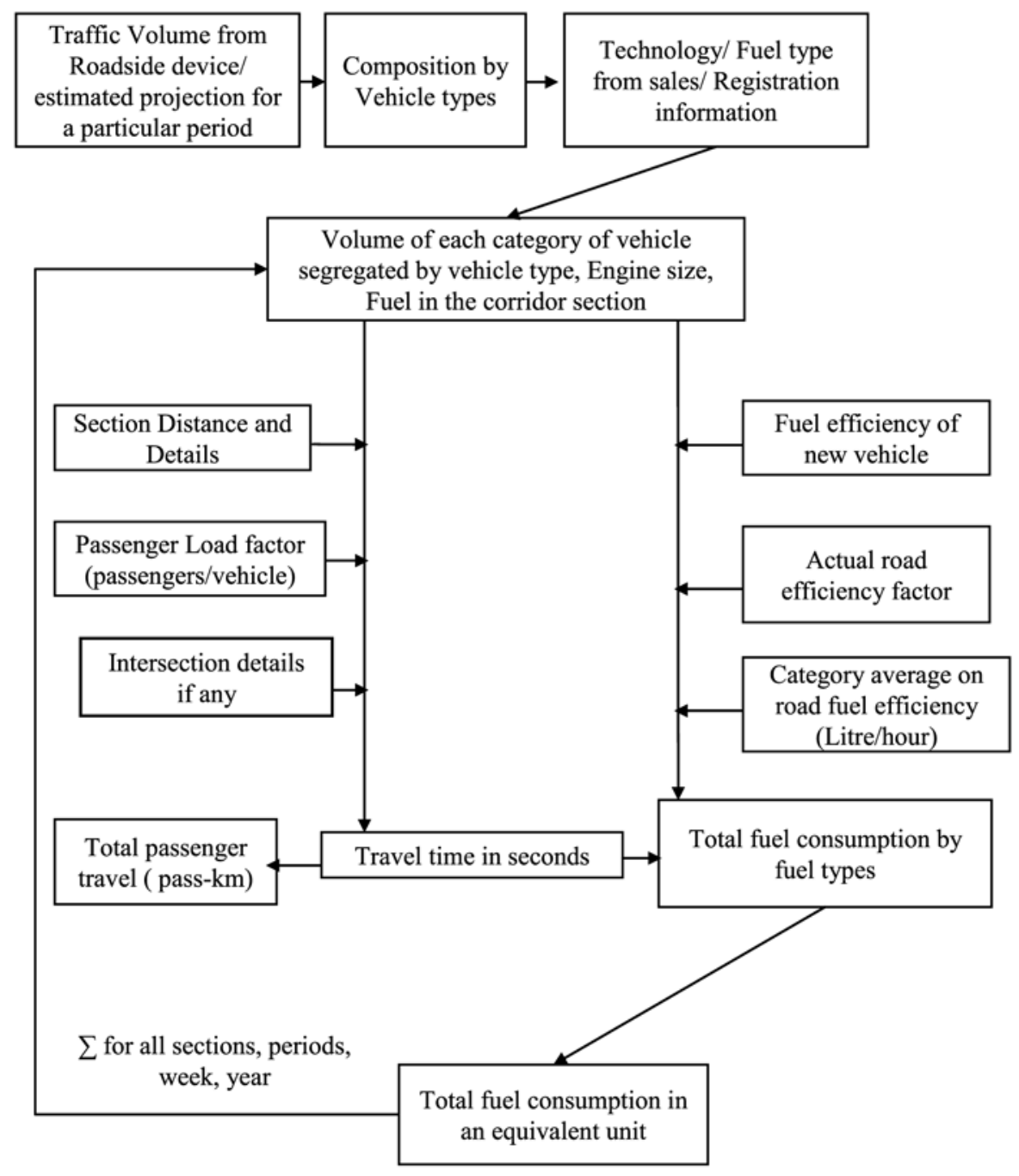

Figure 1. STEP Model Structure

\section{Traffic Data for Model Development}

Traffic data from a series of Automatic Incident Detection (AID) camera stations situated along the Cheras Road corridor were provided by the Urban Transportation Department, Kuala Lumpur City Hall. At each AID station, a video camera and image processing unit are used to record continuously the number of passing vehicles, average vehicle speed, vehicle density, and other parameters averaged 
over three-minute periods for each lane and vehicle class. Three vehicles classes are distinguished according to vehicle length: large, medium, and light category. The data were used to formulate the traffic flow models and estimate the model parameters. Data was available from cameras located at eight positions along the investigated part of the Cheras Road corridor. Figure 2 shows the location of the cameras used in the present project.

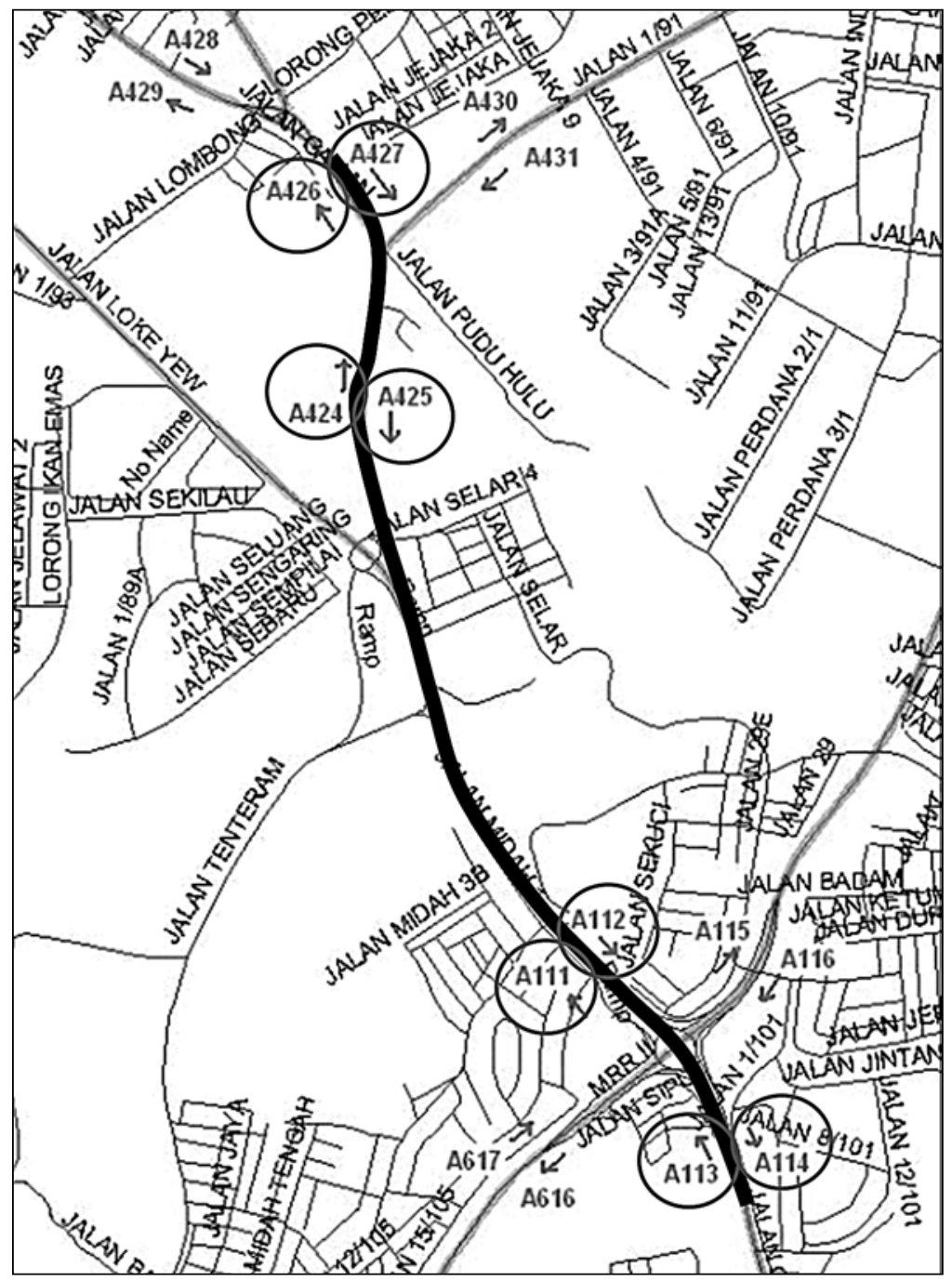

Figure 2. Camera Locations on Cheras Road, Kuala Lumpur 


\section{Traffic Model}

The objective of the traffic model is to estimate an hourly profile of realized vehicle volumes and travel times based on inputs of hourly passenger trip demand and various scenario parameters. The traffic model is actually a two-state model that estimates volumes and travel times separately for stable and unstable flow conditions. A new approach, described here, has been developed to estimate the percentage of time that traffic will reside in an unstable or stable state, based on the typical trip demand for a given period of the day. An expected value for the realized vehicle flow volume is found by summing stable and unstable volumes weighted by the probability of residing in either state. A similar approach is used to find the total travel time.

Stable and Unstable Traffic Flow Models. The distinction between stable and unstable flow is based on the traffic flow speed. When the speed crosses below a threshold value, denoted here as the breakdown speed $\left(s_{b}\right)$, the flow is considered unstable, while it is considered stable for all greater speeds. Figure 3 shows sample observations of speed and volume averaged over three-minute periods for an uninfluenced section of an urban arterial road in Kuala Lumpur. The breakdown speed is set here to $40 \mathrm{kmph}$. The upper portion of the data reveals an inverse relationship between speed and volume; as volume increases, the speed gradually declines. This region is considered stable. The lower portion reveals a region of unstable flow where both speed and volume are reduced due to interactions among a high density of vehicles.

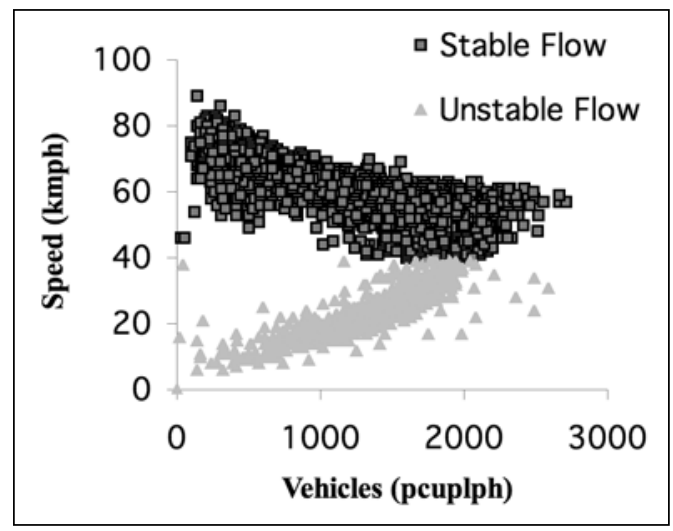

Figure 3. Traffic Volume as Function of Speed 
Classical volume-delay models can be formulated to approximate the relationship between speed and volume in the stable region. The volume-delay model used to represent the traffic flow during stable conditions was the Bureau of Public Roads (BPR) model (Highway Capacity Manual 2000).

$$
t=t_{f}\left(1+\alpha\left(\frac{v}{c}\right)^{\beta}\right)
$$

where:

$t \quad$ is the time required to travel the section length

tf is the free-flow time

$v \quad$ is the number of vehicles (or pcu) per hour

c is the capacity limit for the lane or group of lanes under consideration

The capacity is taken as the 98th percentile of observed volumes for a given section. Model parameters $\alpha$ and $\beta$ are estimated by fitting the model to observed traffic data.

In the unstable region, the relationship between speed and volume becomes much more complex and a direct relationship between volume and speed was not determined. Instead, using a month-long series of three-minute averaged speed and volume observations for different sections, a correlation was found between the stable volumes and the percentage of time that the flow became unstable during the corresponding period. Stable flow and flow breakdown are mutually exclusive. Therefore, if flow breakdown occurred in 25 percent of the observations for a given period, this value $(\% \mathrm{FB}=0.25)$ was then compared to the average of the stable volumes from the remaining 75 percent of the sample. The frequency of flow breakdown for a given period is roughly proportional to the mean stable volume observed over the same period, at least over a range of values for stable volume. A sample plot of stable volume, $v_{s}$, and flow breakdown frequency, $f_{f b}$, is shown for a month-long series of observations on an inbound section of Ipoh Road in Kuala Lumpur in Figure 4. A linear regression line is shown with $R^{2}=0.73$. 


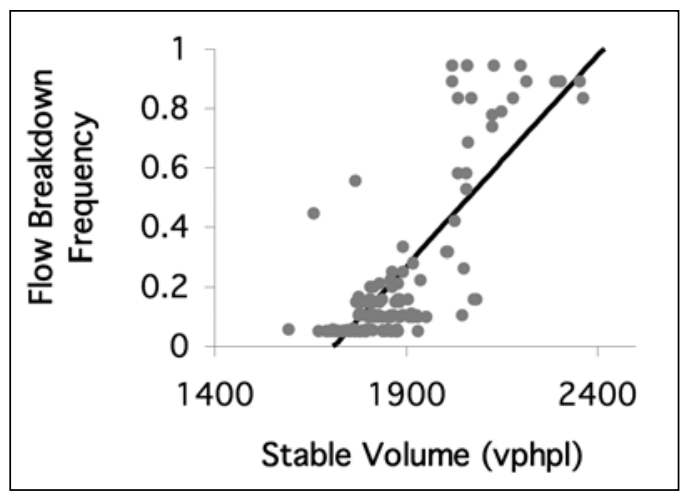

\section{Figure 4. Stable Volume and Flow Breakdown Frequency Sample Plot}

The relationship between stable volume and flow breakdown can be explained by considering the mean stable volume as a proxy for the travel demand. At peak commuting periods, travel demand is at its highest and the traffic occasionally reaches high flow volumes under stable conditions. However, during the same periods, the frequency of flow breakdown is also at its highest. When flow becomes unstable, the speed and volume are reduced drastically, even though demand for that route may be high. By isolating only the stable volume values and comparing these to the frequency of flow breakdown for the same three-minute time period across many days, we are attempting to compare flow breakdown with the level of travel demand, although demand cannot be measured directly.

The relationship between $v_{s}$ and $f_{f b}$ was then characterized by finding the slope and intercept of the regression line, $r$ and $s$, resulting in a linear flow breakdown model as below.

$$
f_{f b}=r v_{s}+s
$$

Utilizing data from the AID camera stations, the stable BPR model and the flow breakdown model have been calibrated for five generalized flow types in the city of Kuala Lumpur; the parameters of which are shown in Table 1. 


\section{Table 1. Parameters for BPR and Proposed Flow Breakdown Models under Various Flow Range Situations}

\begin{tabular}{|l|c|c|c|c|c|c|c|}
\hline & $\begin{array}{c}\text { Free- } \\
\text { Capacity } \\
\text { Flow Type }\end{array}$ & $\begin{array}{c}\text { Flow/hr/lane } \\
\text { Speed } \\
\text { Pmph }\end{array}$ & $\boldsymbol{A}$ & $\beta$ & $\boldsymbol{r}$ & $\boldsymbol{S}$ & $\begin{array}{c}\text { Average } \\
\text { Breakdown } \\
\text { Speed } \\
\text { kmph }\end{array}$ \\
\hline $\begin{array}{l}\text { Uninfluenced } \\
\text { high flow, } \\
\text { suburban }\end{array}$ & 2710 & 89 & 0.722 & 1.045 & 0.058 & 0.016 & 15 \\
\hline $\begin{array}{l}\text { Uninfluenced } \\
\text { flow, } \\
\text { suburban }\end{array}$ & 2010 & 93 & 0.503 & 0.548 & 0.155 & 0.004 & 22.5 \\
\hline $\begin{array}{l}\text { Uninfluenced } \\
\text { flow, urban }\end{array}$ & 1440 & 72 & 0.506 & 0.533 & 0.145 & 0.019 & 17.5 \\
\hline $\begin{array}{l}\text { Medium- } \\
\text { density } \\
\text { traffic signal } \\
\text { vicinity }\end{array}$ & 910 & 51 & 0.774 & 0.454 & 0.637 & -0.067 & 12.5 \\
\hline $\begin{array}{l}\text { High-density } \\
\text { traffic signal } \\
\text { vicinity }\end{array}$ & 700 & 51 & 0.813 & 0.414 & 0.602 & -0.05 & 7.5 \\
\hline
\end{tabular}

\section{Fuel Consumption Model}

Numerous agencies provide standard fuel economy data based on standardized driving cycles for various new vehicles each year. The U.S. Department of Energy and the Environmental Protection Agency jointly publish annual fuel economy statistics using the EPA driving cycle, while the Vehicle Certification Agency of the UK provides a similar annual database using the EC driving cycle. The EPA and EC driving cycles are representative of "typical" driving behavior for city or highway situations. As such they have been developed to estimate fuel consumption at an aggregate level, only differentiating between city and highway driving. They cannot be used to estimate changes in fuel consumption that would occur locally when driving behavior diverges from the standard cycles. One possible solution could be to scale the fuel consumption up or down according to changes in mean trip speed. However, even for trips with the same average speed, one can observe widely different instantaneous speed and acceleration profiles, each resulting in different fuel consumption and emission levels (Rakha et al. 2000). A 
more accurate approach would be to abandon the use of standard driving cycles and estimate the relationship between fuel consumption, speed, and acceleration directly. In a study by Rakha et al. (2000) and Ahn et al. (2002), the authors estimated fuel consumption and emissions levels as a function of instantaneous speed and acceleration based on comprehensive dynamometer tests performed on five light-duty automobiles and three light-duty trucks (West et al. 1997). Averaging the data into a "composite" vehicle, four curves were presented that relate instantaneous fuel consumption to instantaneous vehicle speed for four driving modes, respectively. These are rapid acceleration $\left(1.8 \mathrm{~m} / \mathrm{s}_{2}\right)$, moderate acceleration (0.9 $\left.\mathrm{m} / \mathrm{s}_{2}\right)$, steady speed, and deceleration $\left(-0.9 \mathrm{~m} / \mathrm{s}_{2}\right)$. For the purpose of our present study, we take a weighted average of the four curves according to the frequency that each mode occurs during the EC driving cycle. The EC driving cycle is used as there has not yet been a driving cycle constructed for Malaysian conditions. The resulting single curve, shown in Figure 5, represents the fuel consumption for a given average speed. To calibrate the curve for a specific vehicle type, all values are multiplied by a correction factor, which is the ratio of the average fuel consumption for the vehicle type in question over the fuel consumption for the composite vehicle in the study by Rakha et al. With this approach, the present model can accommodate other vehicle types, which might be available in future.

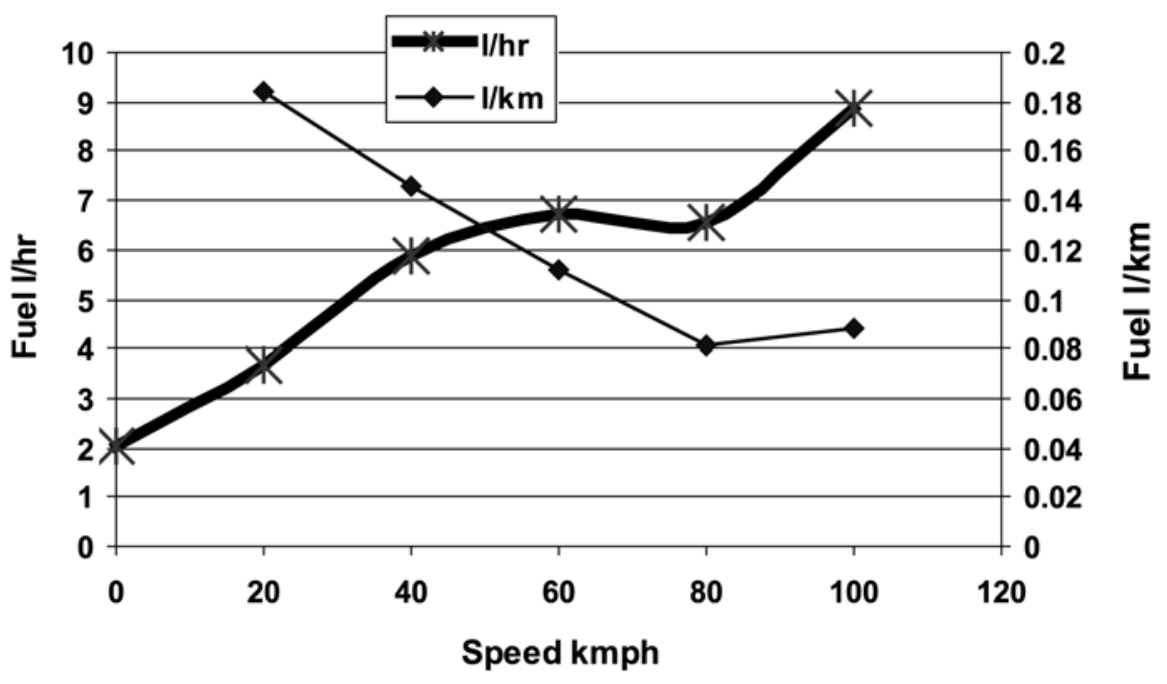

Figure 5. Calibrated Fuel Consumption vs. Speed for Light Vehicles 
By estimating the operating speed from the volume-delay function for stable flow and the mean speed for unstable flow, the fuel consumption can be estimated for the different vehicle types in the stream for all periods of the day.

\section{Model Outputs}

The primary output is the fuel consumption by vehicle category for all sections along the route. Other outputs include operating speed, percent flow breakdown, congestion index (i.e., ratio of a certain travel time period to free-flow travel time), and the amount of diverted demand. The latter is measured in terms of passenger trips.

\section{Model Application}

The Cheras Road corridor of Kuala Lumpur has been chosen as the case study to test the model development. Total length of this corridor, from the intersection of Cheras Road and the Cheras-Kajang Expressway to the Taman Maluri city hub is approximately $11.5 \mathrm{~km}$ in both directions. Along the corridor, typical speed, acceleration, volume, and even type of vehicle may change. The full corridor is therefore divided into sections that are classified according to the five generalized flow types identified in Table 1. The segmentation of the Cheras Road corridor according to section type, length, and lane numbers for each segment are given in Table 2.

\section{Vehicle Fleet Characteristics}

The composition of the present-day Malaysian vehicle fleet is developed from registration and fuel consumption data disaggregated by vehicle type, engine size, and fuel technology (Hossain and Kennedy 2006). The technological aspects of the vehicle fleet are kept constant throughout the planning horizon (i.e., no changes in fuel efficiency) and fuel technology are assumed for any of the scenarios. Typical vehicle characteristics for Malaysian fleet are presented in Table 3.

In future scenarios, the user can define two of the following three sets of values: (1) percentage share of total vehicles by vehicle type, (2) percentage share of total passenger trips by vehicle type, and/or (3) average number of passengers for each vehicle type. If two categories are set, the other will automatically be determined. 


\section{Table 2. Section Characteristics}

\begin{tabular}{|c|l|c|c|c|}
\hline Section & \multicolumn{1}{|c|}{ Flow Type } & $\begin{array}{c}\text { Length } \\
(\mathbf{k m})\end{array}$ & $\begin{array}{c}\text { Fo. of } \\
\text { Lanes }\end{array}$ & $\begin{array}{c}\text { Flow } \\
\text { Speed } \\
\boldsymbol{k m p h}\end{array}$ \\
\hline 1 & $\begin{array}{l}\text { Uninfluenced high- } \\
\text { flow, suburban }\end{array}$ & 2.3 & 4 & 89 \\
\hline 2 & $\begin{array}{l}\text { Uninfluenced flow, } \\
\text { suburban }\end{array}$ & 1.95 & 4 & 93 \\
\hline 3 & $\begin{array}{l}\text { Uninfluenced flow, } \\
\text { urban }\end{array}$ & 1 & 3 & 72 \\
\hline 4 & $\begin{array}{l}\text { Medium-density traffic } \\
\text { signal vicinity }\end{array}$ & 0.35 & 4 & 51 \\
\hline 5 & $\begin{array}{l}\text { Medium-density traffic } \\
\text { signal vicinity }\end{array}$ & 0.35 & 3 & 51 \\
\hline 6 & $\begin{array}{l}\text { Uninfluenced flow, } \\
\text { urban }\end{array}$ & 1 & 3 & 72 \\
\hline 7 & $\begin{array}{l}\text { Uninfluenced flow, } \\
\text { suburban }\end{array}$ & 1.95 & 3 & 93 \\
\hline 8 & $\begin{array}{l}\text { Uninfluenced high- } \\
\text { flow, suburban }\end{array}$ & 2.3 & 4 & 89 \\
\hline
\end{tabular}

\section{Table 3. Typical Vehicle Characteristics for Malaysian Fleet}

\begin{tabular}{|c|c|c|c|c|}
\hline $\begin{array}{l}\text { Vehicle } \\
\text { Type }\end{array}$ & Fuel Type & $\begin{array}{l}\text { Engine } \\
\text { Size } \\
\text { (I) }\end{array}$ & $\begin{array}{c}\text { Fuel } \\
\text { Consumption } \\
(1 / 100 \mathrm{~km})\end{array}$ & $\begin{array}{c}\text { Fraction of } \\
\text { Total } \\
\text { Registered } \\
\text { Vehicles }\end{array}$ \\
\hline \multirow{7}{*}{ Car \& LDV } & \multirow{4}{*}{ Gasoline } & $<1.0$ & 9 & 0.26 \\
\hline & & $1.0-1.5$ & 11 & 0.32 \\
\hline & & $1.5-2.0$ & 12 & 0.24 \\
\hline & & $>2.0$ & 13.5 & 0.051 \\
\hline & Diesel & $>2.0$ & 12.6 & 0.0052 \\
\hline & \multirow{2}{*}{ CNG } & $1.0-1.5$ & 11 & 0.0021 \\
\hline & & $1.5-2.0$ & 12 & 0.00014 \\
\hline \multirow{3}{*}{ MDV "Truck } & Gasoline & $<2.0$ & 15 & 0.066 \\
\hline & \multirow{2}{*}{ Diesel } & $2.0-3.0$ & 17 & 0.0023 \\
\hline & & $>3.0$ & 19 & 0.0093 \\
\hline Truck & Diesel & $>4.0$ & 47 & 0.033 \\
\hline \multirow{3}{*}{ Bus } & Gasoline & $<3.0$ & 50 & 0.00079 \\
\hline & \multirow{2}{*}{ Diesel } & $<3.0$ & 50 & 0.0025 \\
\hline & & $>4.0$ & 55 & 0.0046 \\
\hline
\end{tabular}

*LDV-Light-duty vehicle

${ }^{* *}$ MDV-Medium-duty vehicle 


\section{Sensitivity Analysis}

For calibration of the model as a whole, a sensitivity analysis was performed for trip growth rate. For the base year of 2006 in study area, the trip growth rate was set to 2.73 percent per year. The growth rate in energy consumption, however, exceeded the trip growth rate, due to the influence of increased congestion on fuel consumption. For annual trip growth rates of 2.73 percent, 4 percent, and 5 percent, energy consumption increased by 3.3 percent, 5 percent, and 6.15 percent, respectively, for a typical scenario. The consistent result from the model shows the validity of the model in general.

\section{Scenarios}

Three scenarios have been developed for comparison: business as usual (BAU), bus lane (BL), and bus rapid transit (BRT). The latter two scenarios are differntiated from BAU according to changes in modal share of bus transport, average bus occupancy, and avearage bus traveling speed. The reasoning behind the values chosen for each scenario is described below. For the BL and BRT scenarios, two different cases are examined. First, an existing lane is converted to a restricted lane for buses or BRT [no lane extension case (NLE)]. Alternatively, a new lane is added in each direction for the bus or BRT [lane extension case (LE)]. The base year is set to 2006. All scenarios assume a constant growth rate in trip demand at 2.73 percent per year and are evaluated in 2010 and 2020. The percentage share of total trips made by medium-duty vehicle (MDV) and truck also remain constant at 7 and 4 percent, respectively. Hence, the growth in trip demand by these two modes, which are more likely to be commercially based, is pegged to the overall trip growth.

BAU Scenario. The BAU assumes that no changes are made to the composition of the vehicle fleet over the entire time horizon. In other words, the percentage of vehicles taken up by cars (88.1\%), MDV-truck (7.8\%), trucks (3.3\%), and buses $(0.8 \%)$ remains the same in 2010 and 2020, even though the total number of vehicles increases to satisfy growing trip demand. There is no change in the quality or size of buses, so the average number of passengers per bus remains the same as the initial year (20 pass/bus). There is also no change in the average number of passengers for cars, MDV-truck, or trucks, so the modal trip share also remains constant for all modes (i.e., cars [77\%], MDV-truck [8\%], trucks [4\%], and buses [10.7\%]).

$B L$ Scenario. The BL scenario represents a situation with dedicated bus lanes, but with an inferior level of service compared to a BRT. Passenger comfort, travel 
time, ticketing, and station facilities are all similar to the present-day bus system. Designated bus lanes without physical barriers have been implemented in Kuala Lumpur in the past. These projects have met with very limited success due to nonobservance of designated areas by private vehicles, lack of improvement in other aspects of bus service, and a perceived lack of space to allow continuous bus lanes. We have chosen to represent this type of scenario with a very modest improvement in modal share for buses of 12 percent in 2010 and 15 percent in 2020. These value are intended to represent the impact of a "partial commitment" to bus service improvement and they can be adjusted if more detailed modal share forecasts are available. There is also a slight increase in passengers per bus as compared to BAU due to improved travel times in the dedicated lanes, from 25 per bus in 2010 to 30 per bus in 2020. A peak-hour bus operating speed of $18 \mathrm{kmph}$ and off-peak hour operating speed of $24 \mathrm{kmph}$ are assumed in this scenario based on the current speed situation in the existing bus lanes. However, these values can also be changed by the user to accommodate a different set of assumptions under different situations.

BRT Scenario. In the BRT scenario the level of service of the BRT system is considered to be sufficiently high to justify a large modal shift from private vehicles to BRT. A mix of financial incentives, assistance, regulatory, and enforcement mechanisms can be exercised to create a favorable situation for service improvement and subsequent increase in public transport patronage. A few Southeast Asian cities have experienced significant increases in bus ridership in recent years with such measures. For example, by increasing its control over bus routes, schedules, fares, and overall system design, the Seoul Metropolitan Government has achieved a bus ridership increase as high as 14 percent with no visible impact on metro ridership (Pucher et al. 2005). After introduction of BRT in Jakarta, about 14 percent of car drivers (BAQ Media Team 2006) have shifted to the improved bus service alongside the general increase in bus patronage. Assuming a Malaysian government urban transport policy favoring such public transport patronage, we have chosen to set the BRT modal share to the target value that has been articulated in Malaysia's current five-year plan, which is 30 percent in 2010 and 40 percent in 2020 (EPU 2006), to illustrate the effect that achieving this modal share would have on energy consumption. These modal share values are assumed for this corridor only and not for the entire city-setting high targets for corridor-specific values more likely will be achievable than for a citywide average. Average passengers per bus are also taken as higher in anticipation of the introduction of higher capacity buses, with 40 passengers per bus in 2010 and 50 per bus in 2020. During 
peak-demand periods, articulated buses with a capacity of up to 200 passengers have been considered to be available. A peak-hour bus operating at $24 \mathrm{kmph}$ and an off peak-hour one operating at $32 \mathrm{kmph}$ are assumed in this scenario based on the existing BRT system performance (U.S. FTA 2004).

\section{Results}

\section{Total Consumption by Fuel Type}

Total fuel consumption per week along the corridor was calculated for gasoline, diesel, and natural gas vehicles for the LE case. Quantities of all liquid fuels are provided in liters, while natural gas is in gasoline-equivalent liters. In all scenarios, gasoline consumption dominates all other fuels, as gasoline-powered cars make up the largest proportion of the fleet. Natural gas use is negligible as only a very small percentage of vehicles currently use this fuel.

For BAU, weekly gasoline consumption is initially at 574,000 liters and climbs to 649,000 liters in 2010 and 903,000 liters in 2020. In the BL scenario, gasoline consumption reaches 585,000 liters in 2010 and 722,000 liters in 2020. For the BRT scenario, total gasoline consumption is the lowest at 434,000 liters in 2010 and 464,000 liters in 2020. Therefore, in the BRT case, gasoline consumption has been reduced relative to the BAU case by 33 percent in 2010 and 49 percent in 2020, mainly due to assumed modal shift from car to BRT system.

Diesel consumption, on the other hand, increases as more buses are included in the vehicle fleet. For the BAU case, weekly diesel consumption is initially at 55,000 liters in 2006 and climbs to 62,000 liters in 2010 and 86,000 liters in 2020. In the BL scenario, diesel consumption reaches 59,000 liters in 2010 and 79,000 liters in 2020. For the BRT scenario, total diesel consumption is the highest at 67,000 liters in 2010 and 90,000 liters in 2020. Therefore, the increase in BRT diesel consumption relative to the BAU case is 8 percent in 2010 and 5 percent in 2020. Although the BRT scenario bus numbers are significantly higher than the BAU scenario, there is only a small increase for diesel consumption in 2020 because of the improved operating conditions in the BRT lane.

\section{Total Energy Savings}

Total energy consumption across all fuels is calculated to compare the effectiveness of the different scenarios in terms of energy savings (see Table 4). Measured in ktoe/week, the BAU scenario has an intial energy consumption of $0.52 \mathrm{ktoe}$ in 2006, rising to 0.55 ktoe in 2010 and 0.72 ktoe in 2020 . For the BL scenario, total 
energy consumption is 0.53 ktoe in 2010 and 0.66 ktoe in 2020. With the BRT option, total fuel consumption in the corridor is reduced relative to BAU by 24 percent (for LE and NLE) in 2010 and by about 36 percent (LE) to 40 percent (NLE) in 2020. The maximum possible energy savings are realized by moving from a BAU and NLE scenario to the BRT and LE scenario, reaching 29 percent and 45 percent by 2010 and 2020, respectively.

\section{Table 4. Total Energy (ktoe/week) Savings for Various Scenarios and Cases}

\begin{tabular}{|c|c|c|c|c|}
\hline \multirow{5}{*}{$\begin{array}{l}\text { Lane } \\
\text { Extension } \\
\text { Case (LE) }\end{array}$} & Year & BAU & BL & BRT \\
\hline & 2010 & 0.55 & 0.53 & 0.42 \\
\hline & & & $-4 \%$ & $-24 \%$ \\
\hline & 2020 & 0.72 & 0.66 & 0.46 \\
\hline & & & $-8 \%$ & $-36 \%$ \\
\hline \multirow{5}{*}{$\begin{array}{l}\text { No Lane } \\
\text { Extension } \\
\text { Case (NLE) }\end{array}$} & Year & BAU & BL & BRT \\
\hline & 2010 & 0.59 & 0.57 & 0.45 \\
\hline & & & $-3 \%$ & $-24 \%$ \\
\hline & 2020 & 0.83 & 0.73 & 0.50 \\
\hline & & & $-8 \%$ & $-40 \%$ \\
\hline \multirow{3}{*}{$\begin{array}{l}\text { Improvement } \\
\text { in Energy } \\
\text { Savings Due } \\
\text { to Lane } \\
\text { Extension }\end{array}$} & Year & BAU & BL & BRT \\
\hline & 2010 & $6.8 \%$ & $7 \%$ & $6.7 \%$ \\
\hline & 2020 & $13.2 \%$ & $9.6 \%$ & $8 \%$ \\
\hline \multirow{3}{*}{$\begin{array}{l}\text { Maximum } \\
\text { Possible } \\
\text { Energy } \\
\text { Saving }\end{array}$} & Year & BAU & $\mathrm{BL}$ & BRT \\
\hline & 2010 & & & $29 \%$ ** \\
\hline & 2020 & & & $45 \%^{\star * *}$ \\
\hline
\end{tabular}

** In case of BRT and LE scenario with respect to BAU and NLE scenario.

\section{Conclusions and Recommendations}

The spreadsheet-based model described in this article is a useful tool for estimating energy savings for public transport improvement options and other related transport scenarios. Effects due to congestion have been included in the energy estimates through a new approach at modeling the onset of unstable flow, which otherwise would normally require a more complex microsimulation technique. Implementing a BRT system results in significant improvements in energy efficiency for the urban road corridor in this study. Relative to business as usual, BRT 
reduces the total fuel consumption in the corridor by 24 percent (for both LE and NLE case) in 2010 and about 36 percent (LE case) to 40 percent (NLE case) in 2020. The impact of adding an additional lane is shown to reduce energy consumption within each individual scenario in the range of 7 to 13 percent with the maximum possible reduction with respect to the BAU scenario. This is due to reduced congestion of traffic after addition of a new lane. Demand is assumed to remain constant in the present analysis whether or not a new lane is added. The maximum possible energy savings between any two scenarios are realized by moving from a BAU scenario with no lane extension to the BRT scenario with an additional lane, reaching 29 and 45 percent by 2010 and 2020, respectively. The energy required to construct an additional lane has not been included in this energy analysis. An interesting extension would be to determine the length of time required for energy expended by lane construction to be recovered by fuel savings. This energy payback period could be an additional useful criterion for deciding on road-widening projects.

Although the model can provide output related to operation conditions and fuel consumption under different scenarios, especially future modal split targets set by the user, it has no mechanism for addressing issues in achieving those modal split targets. Even with improved operation and level of service, some control/enforcement measures, like road pricing, parking restriction, and fuel taxation, might also be necessary to achieve the set target for public transport. Also, the model's scope is limited to the boundary of a corridor without considering any influence from parallel or nearby routes. However, for a realistic project feasibilty study, it ultimately comes down to a corridor-level analysis for implementation decisions. In that perspective, this modeling tool can help promote bus transit improvements with its capability of realistic estimations of energy savings for such initiatives.

The present analysis has focused exclusively on energy consumption as an objective for bus improvement initiatives. However, vehicle emissions are another very important criterion for sustainable urban transport; hence, the modeling approach used here should be extended in future work to estimate vehicular emissions from the different vehicle types. Since vehicle-type distribution and speed and volume profiles are already specified by the model, emissions could be determined fairly accurately by including vehicle emission factors. This extension is especially important considering that an expanded bus fleet can potentially damage urban air quality if insufficient pollution control measures are in place. The present model can therefore provide a very useful platform for not only esti- 
mating fuel consumption, but also for testing the corridor-specific impacts of new engine technology on vehicular emissions.

\section{Endnote}

${ }^{1}$ In the current formulation of the STEP model, motorcycles have not been included.

\section{References}

Ahn, K., H. Rakha, A. Trani, and M. V. Aerde. 2002. Estimating fuel consumption and emissions based on instantaneous speeds and accelerations. Journal of Transportation Engineering (April): 182-190.

BAQ Media Team. 2006. Bus rapid transit systems offer effective solution for Asian cities. Accessible at http://www.cleanairnet.org/baq2006/1757/propertyvalue26756.html\#h2_3.

Barth, M., F. An, J. Norbeck, and M. Ross, M. 1996. Modal emissions modeling: A physical approach. Transportation Research Record No. 1520, pp. 81-88, Transportation Research Board, National Academy of Science.

Biggs, D. C., and R. Akcelik. 1986. An energy-related model of instantaneous fuel consumption. Traffic Engineering Control 27(6): 320-325.

Brzezinski, D. J., P. Enns, and C. Hart.1999. Facility-specific speed correction factors. MOBILE6 Stakeholder Review Document (M6.SPD.002). Ann Arbor, MI: U.S. Environmental Protection Agency.

EEA. 2005. EMEP/CORINAIR. Emission inventory guidebook-2005. European Environment Agency, Part B, Group 7-Road Transport.

EPU. 2006. Ninth Malaysia plan 2006-2010. Putrajaya, Malaysia: Economic Planning Unit, Prime Minister's Department.

GDRC (Global Development Research Center). 2007. Sustainable transport: Programs and initiatives. Retrieved on August 14, 2007 from http://www.gdrc. org/uem/sustran/sustran.html.

Graham, C. 2005. The demand performance of bus rapid transit. Journal of Public Transportation 8(1). 
Hensher, D. 2007. Sustainable public transport systems: Moving towards a value for money and network-based approach and away from blind commitment. Transport Policy 14:98-102.

Highway Capacity Manual. 2000.Transportation Research Board.

Hossain, M., and S. Kennedy. 2006. Estimating potential energy savings with efficient public transport alternatives under various traffic scenarios: A spreadsheet based tool for Kuala Lumpur. Sponsored by DANIDA-Economic Planning Unit of Malaysian Government (December).

International Energy Agency (IEA). 2007. [Online] IEA Statistics, accessed June 26, 2007 from International Energy Agency website, http://www.iea.org/Textbase/stats/index.asp.

National Energy Balance, Malaysia (NEB). 2005. Ministry of Energy, Water and Communications, Malaysia.

Ntziachristos, L., and Z. Samaras.2000.COPERT III Computer programme to calculate emissions from road transport Methodology and emission factors (Version 2.1). Report to the European Environmental Agency Topic Center on Air Emissions, Thessaloniki, Greece.

Post, K., J. H. Kent, J. Tomlin, and N. Carruthers. 1984. Fuel consumption and emission modelling by power demand and a comparison with other models. Transportation Research Part A 18(3): 191-213.

Pucher, J., M. H. Kim, and J. Song.2005. Public transport reforms in Seoul: Innovations motivated by funding crisis. Journal of Public Transportation 8.5.

Rakha, H., M. Van Aerde, K. Ahn, and A. A. Trani. 2000. Requirements for evaluating traffic signal control impacts on energy and emissions based on instantaneous speeds and acceleration measurements. TRB 79th Annual Meeting, Washington DC.

Romilly, P. 1999.Substitution of bus for car travel in urban Britain: An economic evaluation of bus and car exhaust emission and other costs. Transportation Research Part D(4):109-125.

SEI (Stockholm Environment Institute). 2006. Long-range energy alternatives planning system. User Guide (March). Retrieved August 13, 2007 from http://www. sei-us.org/default.asp?action $=20$. 
Shahriar, A. Z., and Khan, A. M. 2003. Optimization of travel in bus rapid transitbased multimodal corridor. Journal of Public Transportation 6(2): 87-106.

Tong, H. Y., W. T. Hung, and C. S. Cheung. 2000. On-road motor vehicle emissions and fuel consumption in urban driving conditions. Journal of Air and Waste Management Association 50 (April).

UN ESCAP.2005. Review of developments in transport in Asia and the Pacific 2005. Available at http://www.unescap.org/ttdw/Publications/TPTS_pubs/ pub_2392/pub_2392_fulltext.pdf.

U.S. Department of Energy (U.S. DOE). 2007. Fuel economy guide. U.S. Department of Energy and U.S. Environmental Protection Agency. Retrieved July 11, 2007 from $h t t p: / / w w w$. fueleconomy.gov.

U.S. Federal Transit Administration. 2004. Characteristics of bus rapid transit for decision making. Project No: FTA-VA-26-7222-2004. U.S. Department of Transportation, Office of Research, Demonstration and Innovation.

West, B., R. McGill, J. Hodgson, S. Sluder, and D. Smith. 1997. Development of databased light-duty modal emissions and fuel consumption models. Society of Automotive Engineers, Paper No. 972910: 1274-1280.

\section{About the Authors}

MoAzzem Hossain (moazzem@ce.buet.ac.bd) is a professor of civil engineering at the Bangladesh University of Engineering and Technology. Prior to this position he was an associate professor in the Transportation and Logistics Program at Malaysia University of Science and Technology. His research and consultation interests include transport system simulation, public transport operation, and nonmotorized transport. He has published extensively on the above topics in various journals and conference proceedings. He has developed MIXNETSIM, a mixed-traffic network model for simulating urban road network with heterogeneous traffic. The simulation model has been applied in various developing countries including Bangladesh to simulate use of bus lane and at-grade LRT operation under mixed-traffic situations of developing cities.

SCOTt KenNedY (sknndy@gmail.com) is an associate professor at the Masdar Institute of Science and Technology in Abu Dhabi, United Arab Emirates, and currently a visiting scholar at the Massachusetts Institute of Technology. His research interests are in modeling of energy systems, which include integration of renewable 
Journal of Public Transportation, Vol. 11, No. 3, 2008

energy technologies into constrained electric grids and energy assessments of urban transportation networks. He has also been an active consultant and advisor for energy and environmental policy issues taken up by the Malaysian government. 